\title{
Inclusion of the Generalized Bessel Functions in the Janowski Class
}

\author{
Saiful R. Mondal and Mohammed Al Dhuain \\ Department of Mathematics and Statistics, Collage of Science, King Faisal University, Hofuf, Al-Hasa 31982, Saudi Arabia
}

Correspondence should be addressed to Saiful R. Mondal; smondal@kfu.edu.sa

Received 19 April 2016; Accepted 4 October 2016

Academic Editor: Julien Salomon

Copyright (C) 2016 S. R. Mondal and M. Al Dhuain. This is an open access article distributed under the Creative Commons Attribution License, which permits unrestricted use, distribution, and reproduction in any medium, provided the original work is properly cited.

Sufficient conditions on $A, B, p, b$, and $c$ are determined that will ensure the generalized Bessel function $u_{p, b, c}$ satisfies the subordination $u_{p, b, c}(z) \prec(1+A z) /(1+B z)$. In particular this gives conditions for $(-4 \kappa / c)\left(u_{p, b, c}(z)-1\right), c \neq 0$, to be close-to-convex. Also, conditions for $u_{p, b, c}(z)$ to be Janowski convex and $z u_{p, b, c}(z)$ to be Janowski starlike in the unit disk $\mathbb{D}=\{z \in \mathbb{C}:|z|<1\}$ are obtained.

\section{Introduction}

Let $\mathscr{A}$ denote the class of analytic functions $f$ defined in the open unit disk $\mathbb{D}=\{z:|z|<1\}$ normalized by the conditions $f(0)=0=f^{\prime}(0)-1$. If $f$ and $g$ are analytic in $\mathbb{D}$, then $f$ is subordinate to $g$, written $f(z)<g(z)$, if there is an analytic self-map $w$ of $\mathbb{D}$ satisfying $w(0)=0$ and $f=g \circ w$. For $-1 \leq$ $B<A \leq 1$, let $\mathscr{P}[A, B]$ be the class consisting of normalized analytic functions $p(z)=1+c_{1} z+\cdots$ in $\mathbb{D}$ satisfying

$$
p(z) \prec \frac{1+A z}{1+B z} .
$$

For instance, if $0 \leq \beta<1$, then $\mathscr{P}[1-2 \beta,-1]$ is the class of functions $p(z)=1+c_{1} z+\cdots$ satisfying $\operatorname{Re} p(z)>\beta$ in $\mathbb{D}$.

The class $\mathcal{S}^{*}[A, B]$ of Janowski starlike functions [1] consists of $f \in \mathscr{A}$ satisfying

$$
\frac{z f^{\prime}(z)}{f(z)} \in \mathscr{P}[A, B] .
$$

For $0 \leq \beta<1, \mathcal{S}^{*}[1-2 \beta,-1]:=\mathcal{S}^{*}(\beta)$ is the usual class of starlike functions of order $\beta ; \mathcal{S}^{*}[1-\beta, 0]:=\mathcal{S}_{\beta}^{*}=\{f \in \mathscr{A}$ : $\left.\left|z f^{\prime}(z) / f(z)-1\right|<1-\beta\right\} ;$ and $\mathcal{S}^{*}[\beta,-\beta]:=\mathcal{S}^{*}[\beta]=\{f \in$ $\left.\mathscr{A}:\left|z f^{\prime}(z) / f(z)-1\right|<\beta\left|z f^{\prime}(z) / f(z)+1\right|\right\}$. These classes have been studied, for example, in [2,3]. A function $f \in \mathscr{A}$ is said to be close-to-convex of order $\beta[4,5]$ if $\operatorname{Re}\left(z f^{\prime}(z) / g(z)\right)>\beta$ for some $g \in \mathcal{S}^{*}:=\mathcal{S}^{*}(0)$.
This article studies the generalized Bessel function $u_{p}(z)=u_{p, b, c}(z)$ given by the power series

$$
u_{p}(z)={ }_{0} F_{1}\left(\kappa,-\frac{c}{4} z\right)=\sum_{k=0}^{\infty} \frac{(-1)^{k} c^{k}}{4^{k}(\kappa)_{k}} \frac{z^{k}}{k !},
$$

where $\kappa=p+(b+1) / 2 \neq 0,-1,-2,-3, \ldots$. The function $u_{p}(z)$ is analytic in $\mathbb{D}$ and solution of the differential equation

$$
4 z^{2} u^{\prime \prime}(z)+4 \kappa z u^{\prime}(z)+c z u(z)=0,
$$

if $b, p, c$ in $\mathbb{C}$, such that $\kappa=p+(b+1) / 2 \neq 0,-1,-2,-3, \ldots$, and $z \in \mathbb{D}$. This normalized and generalized Bessel function of the first kind of order $p$ also satisfies the following recurrence relation:

$$
4 \kappa u_{p}^{\prime}(z)=-c u_{p+1}(z),
$$

which is useful tool to study several geometric properties of $u_{p}$. There have been several works [6-11] studying geometric properties of the function $u_{p}(z)$, for example, on its close-toconvexity, starlikeness, and convexity, and radius of starlikeness and convexity.

In Section 2 of this paper, sufficient conditions on $A$, $B, c$, and $\kappa$ are determined that will ensure $u_{p}$ satisfies the subordination $u_{p}(z) \prec(1+A z) /(1+B z)$. It is to be understood 
that a computationally intensive methodology with shrewd manipulations is required to obtain the results in this general framework. The benefits of such general results are that, by judicious choices of the parameters $A$ and $B$, they give rise to several interesting applications, which include extending the results of previous works. Using this subordination result, sufficient conditions are obtained for $(-4 \kappa / c) u^{\prime}(z) \in \mathscr{P}[A, B]$, which next readily gives conditions for $(-4 \kappa / c)\left(u_{p}(z)-1\right)$ to be close-to-convex. Section 3 gives emphasis to the investigation of $u_{p}(z)$ to be Janowski convex as well as of $z u_{p}(z)$ to be Janowski starlike.

The following lemma is needed in sequel.

Lemma 1 (see $[5,12])$. Let $\Omega \subset \mathbb{C}$ and $\Psi: \mathbb{C}^{2} \times \mathbb{D} \rightarrow \mathbb{C}$ satisfy

$$
\Psi(i \rho, \sigma ; z) \notin \Omega
$$

whenever $z \in \mathbb{D}$, $\rho$ is real, and $\sigma \leq-\left(1+\rho^{2}\right) / 2$. If $p$ is analytic in $\mathbb{D}$, with $p(0)=1$, and $\Psi\left(p(z), z p^{\prime}(z) ; z\right) \in \Omega$ for $z \in \mathbb{D}$, then $\operatorname{Re} p(z)>0$ in $\mathbb{D}$.

In the case $\Psi: \mathbb{C}^{3} \times \mathbb{D} \rightarrow \mathbb{C}$, then the condition in Lemma 1 is generalized to

$$
\Psi(i \rho, \sigma, \mu+i v ; z) \notin \Omega \text {. }
$$

$\rho$ is real, $\sigma+\mu \leq 0$, and $\sigma \leq-\left(1+\rho^{2}\right) / 2$.

\section{Close-To-Convexity of Generalized Bessel Functions}

In this section, one main result on the close-to-convexity of the generalized Bessel function with several consequences is discussed in detail.

Theorem 2. Let $-1 \leq B \leq 3-2 \sqrt{2} \approx 0.171573$. Suppose $B<$ $A \leq 1$ and $c, \kappa \in \mathbb{R}$ satisfy

$$
\kappa-1 \geq \frac{(1+B)(1+A)}{4(A-B)}|c| .
$$

$$
\begin{aligned}
& u_{p}(z)=\frac{(1-A)+(1+A) p(z)}{(1-B)+(1+B) p(z)}, \\
& u_{p}^{\prime}(z)=\frac{2(A-B) p^{\prime}(z)}{((1-B)+(1+B) p(z))^{2}}, \\
& u_{p}^{\prime \prime}(z)=\frac{2(A-B)((1-B)+(1+B) p(z)) p^{\prime \prime}(z)-4(1+B)(A-B)\left(p^{\prime}(z)\right)^{2}}{((1-B)+(1+B) p(z))^{3}} .
\end{aligned}
$$

Further let $A, B, \kappa$, and $c$ satisfy either the inequality

$$
\begin{aligned}
(\kappa-1)^{2} & +\frac{(\kappa-1)(1+B)}{(1-B)} \\
& -\left|\frac{(\kappa-1)(A+B)}{2(A-B)} c+\frac{(1+B)^{2}(1+A)}{4(1-B)(A-B)} c\right| \\
& \geq \frac{\left(1-A^{2}\right)\left(1-B^{2}\right)}{16(A-B)^{2}} c^{2}
\end{aligned}
$$

whenever

$$
\begin{aligned}
& \left|2(\kappa-1)(1-B)(A+B) c+(1+B)^{2}(1+A) c\right| \\
& \quad \geq \frac{1}{2}(A-B)(1-B) c^{2}
\end{aligned}
$$

or the inequality

$$
\begin{aligned}
& \left((\kappa-1) \frac{(A+B)}{2(A-B)} c+\frac{(1+B)^{2}(1+A)}{4(1-B)(A-B)} c\right)^{2} \\
& \leq \frac{c^{2}}{4}\left((\kappa-1)^{2}+(\kappa-1) \frac{(1+B)}{1-B}-\frac{(1-A B)^{2}}{16(A-B)^{2}} c^{2}\right)
\end{aligned}
$$

whenever

$$
\begin{aligned}
\left|2(\kappa-1)(1-B)(A+B) c+(1+B)^{2}(1+A) c\right| & \\
& <\frac{1}{2}(A-B)(1-B) c^{2} .
\end{aligned}
$$

If $(1+B) u_{p}(z) \neq(1+A)$ for all $z \in \mathbb{D}$, then $u_{p}(z) \in \mathscr{P}[A, B]$.

Proof. Define the analytic function $p: \mathbb{D} \rightarrow \mathbb{C}$ by

$$
p(z)=-\frac{(1-A)-(1-B) u_{p}(z)}{(1+A)-(1+B) u_{p}(z)}
$$

Then, a computation yields
Thus, using identities (14), the Bessel differential equation (4) can be rewritten as

$$
z^{2} p^{\prime \prime}(z)-\frac{2(1+B)}{(1-B)+(1+B) p(z)}\left(z p^{\prime}(z)\right)^{2}
$$


Assume $\Omega=\{0\}$, and define $\Psi(r, s, t ; z)$ by

$\Psi(r, s, t ; z)$

$$
\begin{aligned}
:= & t-\frac{2(1+B)}{(1-B)+(1+B) r} s^{2}+\kappa s \\
& +\frac{((1-B)+(1+B) r)((1-A)+(1+A) r)}{8(A-B)} c z .
\end{aligned}
$$

It follows from (15) that $\Psi\left(p(z), z p^{\prime}(z), z^{2} p^{\prime \prime}(z) ; z\right) \in \Omega$. To ensure $\operatorname{Re} p(z)>0$ for $z \in \mathbb{D}$, from Lemma 1 , it is enough to establish $\operatorname{Re} \Psi(i \rho, \sigma, \mu+i v ; z)<0$ in $\mathbb{D}$ for any real $\rho, \sigma \leq$ $-\left(1+\rho^{2}\right) / 2$, and $\sigma+\mu \leq 0$.

With $z=x+i y \in \mathbb{D}$ in (16), a computation yields

$\operatorname{Re} \Psi(i \rho, \sigma, \mu+i v ; z)$

$$
\begin{aligned}
= & \mu-\frac{2\left(1-B^{2}\right)}{(1-B)^{2}+(1+B)^{2} \rho^{2}} \sigma^{2}+\kappa \sigma \\
& -\frac{\rho(1-A B)}{4(A-B)} c y \\
& +\frac{(1-B)(1-A)-(1+B)(1+A) \rho^{2}}{8(A-B)} c x .
\end{aligned}
$$

Since $\sigma \leq-\left(1+\rho^{2}\right) / 2$ and $B \in[-1,3-2 \sqrt{2}]$,

$$
\begin{aligned}
& \frac{2\left(1-B^{2}\right)}{(1-B)^{2}+(1+B)^{2} \rho^{2}} \sigma^{2} \\
& \geq \frac{2\left(1-B^{2}\right)}{(1-B)^{2}+(1+B)^{2} \rho^{2}} \frac{\left(1+\rho^{2}\right)^{2}}{4} \geq \frac{1+B}{2(1-B)} .
\end{aligned}
$$

Thus

$$
\begin{aligned}
\operatorname{Re} \Psi(i \rho, \sigma, \mu+i v ; z) & \\
\leq & (\kappa-1) \sigma-\frac{1+B}{2(1-B)}-\frac{\rho(1-A B)}{4(A-B)} c y \\
& +\frac{(1-B)(1-A)-(1+B)(1+A) \rho^{2}}{8(A-B)} c x \\
\leq & -\frac{1}{2}(\kappa-1)\left(1+\rho^{2}\right)-\frac{1+B}{2(1-B)}-\frac{\rho(1-A B)}{4(A-B)} c y \\
& +\frac{(1-B)(1-A)-(1+B)(1+A) \rho^{2}}{8(A-B)} c x \\
= & p_{1} \rho^{2}+q_{1} \rho+r_{1}:=Q(\rho),
\end{aligned}
$$

where

$$
\begin{aligned}
& p_{1}=-\frac{1}{2}(\kappa-1)-\frac{(1+B)(1+A) c x}{8(A-B)}, \\
& q_{1}=-\frac{1-A B}{4(A-B)} c y, \\
& r_{1}=-\frac{1}{2}(\kappa-1)+\frac{(1-B)(1-A)}{8(A-B)} c x-\frac{1+B}{2(1-B)} .
\end{aligned}
$$

Since $\max _{\rho \in \mathbb{R}}\left\{p_{1} \rho^{2}+q_{1} \rho+r_{1}\right\}=\left(4 p_{1} r_{1}-q_{1}^{2}\right) /\left(4 p_{1}\right)$ for $p_{1}<0$, it is clear that $Q(\rho)<0$ when

$$
\begin{aligned}
& \frac{(1-A B)^{2}}{16(A-B)^{2}} c^{2} y^{2} \\
& \quad<4\left(-\frac{1}{2}(\kappa-1)-\frac{(1+B)(1+A)}{8(A-B)} c x\right) \\
& \quad\left(-\frac{1}{2}(\kappa-1)+\frac{(1-B)(1-A)}{8(A-B)} c x-\frac{1+B}{2(1-B)}\right),
\end{aligned}
$$

with $|x|,|y|<1$. As $y^{2}<1-x^{2}$, the above condition holds whenever

$$
\begin{aligned}
& \frac{(1-A B)^{2} c^{2}}{16(A-B)^{2}}\left(1-x^{2}\right) \\
& \quad \leq\left((\kappa-1)+\frac{(1+B)(1+A) c x}{4(A-B)}\right) \\
& \quad\left((\kappa-1)-\frac{(1-B)(1-A)}{4(A-B)} c x+\frac{1+B}{1-B}\right),
\end{aligned}
$$

that is, when

$$
\begin{aligned}
\frac{c^{2}}{16} x^{2} & +\left((\kappa-1) \frac{(A+B)}{2(A-B)} c+\frac{(1+B)^{2}(1+A)}{4(1-B)(A-B)} c\right) x \\
& +(\kappa-1)^{2}+(\kappa-1) \frac{1+B}{1-B}-\frac{(1-A B)^{2}}{16(A-B)^{2}} c^{2} \geq 0 .
\end{aligned}
$$

To establish inequality (24), consider the polynomial $R$ given by

$$
R(x):=m x^{2}+n x+r, \quad|x|<1,
$$

where

$$
\begin{aligned}
& m:=\frac{c^{2}}{16}, \\
& n:=(\kappa-1) \frac{(A+B)}{2(A-B)} c+\frac{(1+B)^{2}(1+A)}{4(1-B)(A-B)} c, \\
& r:=(\kappa-1)^{2}+(\kappa-1) \frac{1+B}{1-B}-\frac{(1-A B)^{2}}{16(A-B)^{2}} c^{2} .
\end{aligned}
$$


Constraint (10) yields $|n| \geq 2|m|$, and thus $R(x) \geq m+r-|n|$. Now inequality $(9)$ readily implies that

$$
\begin{aligned}
R(x) \geq & m+r-|n| \\
= & \frac{c^{2}}{16}+(\kappa-1)^{2}+(\kappa-1) \frac{1+B}{1-B} \\
& -\frac{(1-A B)^{2}}{16(A-B)^{2}} c^{2} \\
& -\left|(\kappa-1) \frac{(A+B)}{2(A-B)} c+\frac{(1+B)^{2}(1+A)}{4(1-B)(A-B)} c\right| \\
= & (\kappa-1)^{2}+(\kappa-1) \frac{(1+B)}{1-B} \\
& -\left|(\kappa-1) \frac{(A+B)}{2(A-B)} c+\frac{(1+B)^{2}(1+A)}{4(1-B)(A-B)} c\right| \\
& -\frac{\left(1-A^{2}\right)\left(1-B^{2}\right)}{16(A-B)^{2}} c^{2} \geq 0 .
\end{aligned}
$$

Now consider the case of constraint (12), which is equivalent to $|n|<2 m$. Then the minimum of $R$ occurs at $x=-n /(2 m)$, and (11) yields

$$
R(x) \geq \frac{4 m r-n^{2}}{4 m} \geq 0 .
$$

Evidently $\Psi$ satisfies the hypothesis of Lemma 1, and thus $\operatorname{Re} p(z)>0$; that is,

$$
-\frac{(1-A)-(1-B) u_{p}(z)}{(1+A)-(1+B) u_{p}(z)} \prec \frac{1+z}{1-z} .
$$

Hence there exists an analytic self-map $w$ of $\mathbb{D}$ with $w(0)=0$ such that

$$
-\frac{(1-A)-(1-B) u_{p}(z)}{(1+A)-(1+B) u_{p}(z)}=\frac{1+w(z)}{1-w(z)}
$$

which implies that $u_{p}(z) \prec(1+A z) /(1+B z)$.

Theorem 2 gives rise to simple conditions on $c$ and $\kappa$ to ensure $u_{p}(z)$ maps $\mathbb{D}$ into a half plane.

Corollary 3. Let $c \leq 0$ and $\kappa \geq \max \left\{5 / 4,1+c^{2} / 2\right\}$. Then $\operatorname{Re} u_{p}(z)>c /(c-1)$.

Proof. Choose $A=-(c+1) /(c-1)$ and $B=-1$ in Theorem 2. Then condition (8) is equivalent to $\kappa \geq 1$ and (10) reduces to $\kappa \geq 5 / 4$, and clearly both hold for $\kappa \geq \max \left\{5 / 4,1+c^{2} / 2\right\}$. The proof will complete if hypothesis (9) holds; that is,

$$
(\kappa-1)^{2} \geq \frac{1}{2}(\kappa-1) c^{2} .
$$

Since $\kappa \geq 1+c^{2} / 2$, it follows that

$$
(\kappa-1)^{2}-\frac{1}{2}(\kappa-1) c^{2}=(\kappa-1)\left(\kappa-1-\frac{c^{2}}{2}\right) \geq 0,
$$

which establishes (31).
Corollary 4. Let $c \in \mathbb{R}$. Then if $\kappa \geq|c| / 2$, then $\operatorname{Re} u_{p}(z)>$ $1 / 2$.

Proof. Put $A=0$ and $B=-1$ in Theorem 2. Condition (8) reduces to $\kappa \geq 1$, which holds in all cases. It is sufficient to establish conditions (10) and (9) or, equivalently,

$$
\begin{aligned}
4(\kappa-1)-|c| & \geq 0, \\
(\kappa-1)^{2}-\frac{1}{2}(\kappa-1)|c| & \geq 0 .
\end{aligned}
$$

The hypothesis $\kappa-1 \geq|c| / 2$ implies that $4(\kappa-1)-|c| \geq|c| \geq 0$ and $(\kappa-1)^{2}-(1 / 2)(\kappa-1)|c| \geq(\kappa-1)(\kappa-1-|c| / 2) \geq 0$.

Next theorem gives the sufficient condition for close-toconvexity when $B \geq 3-2 \sqrt{2}$.

Theorem 5. Let $3-2 \sqrt{2} \leq B<A \leq 1$ and $c, \kappa \in \mathbb{R}$ satisfy

$$
\kappa-1 \geq \frac{(1+B)(1+A)}{4(A-B)}|c| .
$$

Suppose $A, B$, $\kappa$, and $c$ satisfy either the inequality

$$
\begin{aligned}
& (\kappa-1)^{2}-16(\kappa-1) \frac{B(1-B)}{(1+B)^{3}} \\
& \quad-\left|\frac{(\kappa-1)(A+B)}{2(A-B)} c+\frac{4 B\left(1-B^{2}\right)(1+A)}{(1+B)^{3}(A-B)} c\right| \\
& \geq \frac{\left(1-A^{2}\right)\left(1-B^{2}\right)}{16(A-B)^{2}} c^{2}
\end{aligned}
$$

whenever

$$
\begin{aligned}
& \left|(\kappa-1)(1+B)^{3}(A+B) c+8 B\left(1-B^{2}\right)(1+A) c\right| \\
& \quad \geq \frac{c^{2}}{4}(A-B)(1+B)^{3}
\end{aligned}
$$

or the inequality

$$
\begin{aligned}
& \left((\kappa-1) \frac{(A+B)}{2(A-B)} c+\frac{4 B\left(1-B^{2}\right)(1+A)}{(1+B)^{3}(A-B)} c\right)^{2} \\
& \leq \frac{c^{2}}{4}\left((\kappa-1)^{2}+16(\kappa-1) \frac{B(1-B)}{(1+B)^{3}}\right. \\
& \left.-\frac{(1-A B)^{2}}{16(A-B)^{2}} c^{2}\right)
\end{aligned}
$$

whenever

$$
\begin{aligned}
& \left|\left((\kappa-1)(A+B)(1+B)^{3}+8 B\left(1-B^{2}\right)(1+A)\right) c\right| \\
& \quad<\frac{c^{2}}{4}(A-B)(1+B)^{3} .
\end{aligned}
$$

If $(1+B) u_{p}(z) \neq(1+A)$ for all $z \in \mathbb{D}$, then $u_{p}(z) \in \mathscr{P}[A, B]$. 
Proof. First, proceed similarly to the proof of Theorem 2 and derive the expression of $\operatorname{Re} \Psi(i \rho, \sigma, \mu+i v ; z)$ as given in (17). Now, for $\sigma \leq-\left(1+\rho^{2}\right) / 2, \rho \in \mathbb{R}$, and $B \geq 3-2 \sqrt{2}$,

$$
\begin{aligned}
& \frac{2\left(1-B^{2}\right)}{(1-B)^{2}+(1+B)^{2} \rho^{2}} \sigma^{2} \\
& \geq \frac{2\left(1-B^{2}\right)}{(1-B)^{2}+(1+B)^{2} \rho^{2}} \frac{\left(1+\rho^{2}\right)^{2}}{4} \geq \frac{8 B(1-B)}{(1+B)^{3}},
\end{aligned}
$$

and then, with $z=x+i y \in \mathbb{D}$ and $\mu+\sigma<0$, it follows that

$$
\begin{aligned}
\operatorname{Re} \Psi(i \rho, \sigma, \mu+i v ; z) \leq & -\frac{1}{2}(\kappa-1)\left(1+\rho^{2}\right) \\
& -\frac{(1+B)(1+A) \rho^{2}}{8(A-B)} c x \\
& -\frac{\rho(1-A B)}{4(A-B)} c y \\
& +\frac{(1-B)(1-A)}{8(A-B)} c x \\
& -\frac{8 B(1-B)}{(1+B)^{3}} \\
= & p_{2} \rho^{2}+q_{2} \rho+r_{2}:=Q_{1}(\rho),
\end{aligned}
$$

where

$$
\begin{aligned}
& p_{2}=-\frac{1}{2}(\kappa-1)-\frac{(1+B)(1+A)}{8(A-B)} c x, \\
& q_{2}=-\frac{(1-A B) c y}{4(A-B)}, \\
& r_{2}=-\frac{1}{2}(\kappa-1)+\frac{(1-B)(1-A)}{8(A-B)} c x-\frac{8 B(1-B)}{(1+B)^{3}} .
\end{aligned}
$$

Observe that inequality (34) implies that $p_{2}<0$. Thus $Q_{1}(\rho)<0$ for all $\rho \in \mathbb{R}$ provided $q_{2}^{2} \leq 4 p_{2} r_{2}$; that is, for $|x|,|y|<1$,

$$
\begin{gathered}
\frac{(1-A B)^{2}}{16(A-B)^{2}} c^{2} y^{2} \leq\left((\kappa-1)+\frac{(1+B)(1+A)}{4(A-B)} c x\right) \\
\cdot\left((\kappa-1)-\frac{(1-B)(1-A)}{4(A-B)} c x+\frac{16 B(1-B)}{(1+B)^{3}}\right) .
\end{gathered}
$$

With $y^{2}<1-x^{2}$, it is enough to show, for $|x|<1$,

$$
\begin{aligned}
& \frac{(1-A B)^{2}}{16(A-B)^{2}} c^{2}\left(1-x^{2}\right) \\
& \quad \leq\left((\kappa-1)+\frac{(1+B)(1+A)}{4(A-B)} c x\right) \\
& \quad \cdot\left((\kappa-1)-\frac{(1-B)(1-A)}{4(A-B)} c x+\frac{16 B(1-B)}{(1+B)^{3}}\right),
\end{aligned}
$$

which is equivalent to

$$
R_{1}(x):=m_{1} x^{2}+n_{1} x+r_{1} \geq 0,
$$

where

$$
\begin{aligned}
& m_{1}:=\frac{c^{2}}{16}, \\
& n_{1} \\
& \quad:=\left((\kappa-1)\left(\frac{c(A+B)}{2(A-B)}\right)+\frac{4 B\left(1-B^{2}\right)(1+A)}{(A-B)(1+B)^{3}} c\right), \\
& r_{1}:=(\kappa-1)^{2}-(\kappa-1) \frac{16 B(1-B)}{(1+B)^{3}}-\frac{c^{2}(1-A B)^{2}}{16(A-B)^{2}} .
\end{aligned}
$$

If (36) holds, then $\left|n_{1}\right| \geq 2\left|m_{1}\right|$ and $R_{1}(x) \geq m_{1}+r_{1}-\left|n_{1}\right|$, which is nonnegative from (35). On the other hand, if (38) holds, then $\left|n_{1}\right|<2\left|m_{1}\right|, R_{1}(x) \geq\left(4 m_{1} r_{1}-n_{1}^{2}\right) / 4 m_{1}$, and (37) implies $R_{1}(x) \geq 0$. Either case establishes (44).

Theorem 6. Let $-1 \leq B \leq 3-2 \sqrt{2} \approx 0.171573$. Suppose $B<A \leq 1$ and $c, \kappa \in \mathbb{R}$ with $c \neq 0$ satisfying

$$
\kappa \geq \frac{(1+B)(1+A)}{4(A-B)}|c| .
$$

Further let $A, B, \kappa$, and $c$ satisfy either

$$
\begin{aligned}
\kappa^{2}+ & \kappa \frac{1+B}{1-B}-\left|\kappa \frac{(A+B)}{2(A-B)} c+\frac{(1+B)^{2}(1+A)}{4(1-B)(A-B)} c\right| \\
\geq & \frac{\left(1-A^{2}\right)\left(1-B^{2}\right)}{16(A-B)^{2}} c^{2}
\end{aligned}
$$

whenever

$$
\begin{aligned}
& \left|2 \kappa(1-B)(A+B) c+(1+B)^{2}(1+A) c\right| \\
& \quad \geq \frac{1}{2}(A-B)(1-B) c^{2}
\end{aligned}
$$

or the inequality

$$
\begin{aligned}
& \left(\kappa \frac{(A+B)}{2(A-B)}+\frac{(1+B)^{2}(1+A)}{4(1-B)(A-B)} c\right)^{2} \\
& \quad \leq \frac{c^{2}}{4}\left(\kappa^{2}+\frac{\kappa(1+B)}{1-B}-\frac{(1-A B)^{2}}{16(A-B)^{2}} c^{2}\right)
\end{aligned}
$$

when

$$
\begin{aligned}
\mid 2 \kappa & (1-B)(A+B) c+(1+B)^{2}(1+A) c \mid \\
& <\frac{1}{2}(A-B)(1-B) c^{2} .
\end{aligned}
$$

If $(1+B) u_{p}(z) \neq(1+A)$ for all $z \in \mathbb{D}$, then $(-4 \kappa / c) u_{p}^{\prime}(z) \epsilon$ $\mathscr{P}[A, B]$. 
Theorem 7. Let $3-2 \sqrt{2}<B<A \leq 1$. Suppose $c, \kappa \in \mathbb{R}$ and $a \neq 0$, such that

$$
\kappa \geq \frac{(1+B)(1+A)}{4(A-B)}|c| .
$$

Suppose $A, B, \kappa$, and c satisfy either

$$
\begin{aligned}
\kappa^{2}+ & 16 \kappa \frac{B(1-B)}{(1+B)^{3}} \\
& -\left|\kappa \frac{(A+B)}{2(A-B)} c+\frac{4 B\left(1-B^{2}\right)(1+A)}{(1+B)^{3}(A-B)} c\right| \\
& \geq \frac{\left(1-A^{2}\right)\left(1-B^{2}\right)}{16(A-B)^{2}} c^{2}
\end{aligned}
$$

whenever

$$
\begin{gathered}
\left|\kappa(1+B)^{3}(A+B) c+8 B\left(1-B^{2}\right)(1+A) c\right| \\
\geq \frac{c^{2}}{4}(A-B)(1+B)^{3}
\end{gathered}
$$

or the inequality

$$
\begin{aligned}
& \left(\kappa \frac{(A+B)}{2(A-B)} c+\frac{4 B\left(1-B^{2}\right)(1+A)}{(1+B)^{3}(A-B)}\right)^{2} \\
& \leq \frac{c^{2}}{4}\left(\kappa^{2}+\frac{16 \kappa B(1-B)}{(1+B)^{3}}-\frac{(1-A B)^{2}}{16(A-B)^{2}} c^{2}\right)
\end{aligned}
$$

when

$$
\begin{aligned}
\mid 2 \kappa & (1+B)^{3}(A+B) c+8 B\left(1-B^{2}\right)(1+A) c \mid \\
& <\frac{c^{2}}{4}(A-B)(1+B)^{3} .
\end{aligned}
$$

If $(1+B) u_{p}(z) \neq(1+A)$ for all $z \in \mathbb{D}$, then $(-4 \kappa / c) u_{p}^{\prime}(z) \epsilon$ $\mathscr{P}[A, B]$.

Corollary 8. Let $c \leq-1$ and

$$
\kappa \geq \max \left\{\frac{c(c+1)}{2}, \frac{c}{2(c+1)}\right\} .
$$

Then $(-4 \kappa / c)\left(u_{p}(z)-1\right)$ is close-to-convex of order $(c+1) / c$ with respect to the identity function.

Corollary 9. Let $c$ be a nonzero real number and $\kappa \geq|c| / 2$. Then

$$
\operatorname{Re}\left(-\frac{4 \kappa}{c}\right) u_{p}^{\prime}(z)>\frac{1}{2}
$$

\section{Janowski Starlikeness of Generalized Bessel Functions}

This section contributes to finding conditions to ensure a normalized and generalized Bessel function $z u_{p}(z)$ in the class of Janowski starlike functions. For this purpose, first sufficient conditions for $u_{p}(z)$ to be Janowski convex are determined, and then an application of relation (5) yields conditions for $z u_{p}(z) \in \mathcal{S}^{*}[A, B]$.

Theorem 10. Let $c, \kappa \in \mathbb{R}$ be such that $(A-B) u_{p}^{\prime}(z) \neq(1+$ $B) z u_{p}^{\prime \prime}(z)$ for all $z \in \mathbb{D}$ and $-1 \leq B<A \leq 1$. Suppose

$$
\kappa(1+B) \geq \frac{(1+B)^{2}}{4(A-B)}|c|-(1+A-B) .
$$

Further let $A, B, \kappa$, and c satisfy

$$
\begin{aligned}
&(1+A-B+\kappa(1+B))(1-A+B+\kappa(1-B)) \\
& \geq \frac{\left(1-B^{2}\right)^{2}}{16(A-B)^{2}} c^{2} \\
&+\left|\frac{-A\left(B^{2}+1\right)+B\left(3+B^{2}\right)+\left(1-B^{2}\right) B \kappa c}{2(A-B)}\right| .
\end{aligned}
$$

If $0 \notin u_{p}^{\prime}(\mathbb{D})$ and $0 \notin u_{p}^{\prime \prime}(\mathbb{D})$, then

$$
1+\frac{z u_{p}^{\prime \prime}(z)}{u_{p}^{\prime}(z)} \prec \frac{1+A z}{1+B z} .
$$

Proof. Define an analytic function $p: \mathbb{D} \rightarrow \mathbb{C}$ by

$$
p(z):=\frac{(A-B) u_{p}^{\prime}(z)+(1-B) z u_{p}^{\prime \prime}(z)}{(A-B) u_{p}^{\prime}(z)-(1+B) z u_{p}^{\prime \prime}(z)}
$$

Then

$$
\begin{aligned}
& \frac{z u_{p}^{\prime \prime}(z)}{u_{p}^{\prime}(z)}=\frac{(A-B)(p(z)-1)}{(p(z)+1)+B(p(z)-1)}, \\
& \frac{z^{2} u_{p}^{\prime \prime \prime}(z)+z u_{p}^{\prime \prime}(z)}{z u_{p}^{\prime \prime}(z)}-\frac{z u_{p}^{\prime \prime}(z)}{u_{p}^{\prime}(z)} \\
& =\frac{z p^{\prime}(z)}{(p(z)-1)}-\frac{(1+B) z p^{\prime}(z)}{(p(z)+1)+B(p(z)-1)} \\
& =\frac{z p^{\prime}(z)((p(z)+1)+B(p(z)-1)-(1+B)(p(z)-1))}{(p(z)-1)((p(z)+1)+B(p(z)-1))} .
\end{aligned}
$$

A rearrangement of (63) yields

$$
\begin{aligned}
\frac{z u_{p}^{\prime \prime \prime}(z)}{u_{p}^{\prime \prime}(z)}= & \frac{2 z p^{\prime}(z)}{(p(z)-1)((p(z)+1)+B(p(z)-1))} \\
& -1+\frac{z u_{p}^{\prime \prime}(z)}{u_{p}^{\prime}(z)} .
\end{aligned}
$$


Thus,

$$
\begin{gathered}
\left(\frac{z u_{p}^{\prime \prime \prime}(z)}{u_{p}^{\prime \prime}(z)}\right)\left(\frac{z u_{p}^{\prime \prime}(z)}{u_{p}^{\prime}(z)}\right) \\
=\frac{2(A-B)(p(z)-1) z p^{\prime}(z)}{(p(z)-1)((p(z)+1)+B(p(z)-1))^{2}} \\
\quad-\frac{(A-B)(p(z)-1)}{(p(z)+1)+B(p(z)-1)} \\
+\frac{(A-B)^{2}(p(z)-1)^{2}}{((p(z)+1)+B(p(z)-1))^{2}} .
\end{gathered}
$$

Now a differentiation of (4) leads to

$$
4 z^{2} u_{p}^{\prime \prime \prime}(z)+4(\kappa+1) z u_{p}^{\prime \prime}(z)+c z u_{p}^{\prime}(z)=0
$$

which gives

$$
\left(\frac{z u_{p}^{\prime \prime \prime}(z)}{u_{p}^{\prime \prime}(z)}\right)\left(\frac{z u_{p}^{\prime \prime}(z)}{u_{p}^{\prime}(z)}\right)+(\kappa+1) \frac{z u_{p}^{\prime \prime}(z)}{u_{p}^{\prime}(z)}+\frac{c}{4} z=0
$$

Using (62) and (65), (67) yields

$$
\begin{aligned}
& \frac{2(A-B) z p^{\prime}(z)}{((p(z)+1)+B(p(z)-1))^{2}} \\
& \quad+\frac{(A-B)^{2}(p(z)-1)^{2}}{((p(z)+1)+B(p(z)-1))^{2}} \\
& \quad+\frac{(A-B)(p(z)-1) \kappa}{(p(z)+1)+B(p(z)-1)}+\frac{c}{4} z=0,
\end{aligned}
$$

and equivalently

$$
\begin{aligned}
& z p^{\prime}(z) \\
& +\left(\frac{A-B}{2}+\frac{\kappa(1+B)}{2}+\frac{c z(1+B)^{2}}{8(A-B)}\right)(p(z))^{2} \\
& +\left(A-B+\kappa B-\frac{c\left(1-B^{2}\right)}{4(A-B)} z\right) p(z) \\
& +\left(\frac{A-B}{2}-\frac{\kappa(1-B)}{2}+\frac{c z(1-B)^{2}}{8(A-B)}\right)=0 .
\end{aligned}
$$

Define

$$
\begin{aligned}
\Psi\left(p(z), z p^{\prime}(z), z\right):= & z p^{\prime}(z)+F_{1}(p(z))^{2} \\
& +F_{2} p(z)+F_{3},
\end{aligned}
$$

where

$$
\begin{aligned}
& F_{1}=\frac{(A-B)}{2}+\frac{\kappa(1+B)}{2}+\frac{c z(1+B)^{2}}{8(A-B)}, \\
& F_{2}=-(A-B)-\kappa B+\frac{c\left(1-B^{2}\right)}{4(A-B)} z, \\
& F_{3}=\frac{(A-B)}{2}-\frac{\kappa(1-B)}{2}+\frac{c z(1-B)^{2}}{8(A-B)} .
\end{aligned}
$$

Thus, (69) yields $\Psi\left(p(z), z p^{\prime}(z), z\right) \in \Omega=\{0\}$. Now, with $z=x+i y \in \mathbb{D}$, let

$$
\begin{aligned}
G_{1} & :=\operatorname{Re}\left(F_{1}\right)=\frac{A-B}{2}+\frac{\kappa(1+B)}{2}+\frac{c x(1+B)^{2}}{8(A-B)} \\
& =\frac{1}{2}\left(A-B+\kappa(1+B)+\frac{c x(1+B)^{2}}{4(A-B)}\right) \\
G_{2} & :=\operatorname{Re}\left(i F_{2}\right)=-\frac{c\left(1-B^{2}\right)}{4(A-B)} y, \\
G_{3} & :=\operatorname{Re}\left(F_{3}\right)=\frac{A-B}{2}-\kappa \frac{1-B}{2}+\frac{c x(1-B)^{2}}{8(A-B)} \\
& =\frac{1}{2}\left(A-B-\kappa(1-B)+\frac{c(1-B)^{2}}{4(A-B)} x\right) .
\end{aligned}
$$

For $\sigma \leq-\left(1+\rho^{2}\right) / 2, \rho \in \mathbb{R}$,

$$
\begin{aligned}
\operatorname{Re} \Psi(i \rho, \sigma, z) & =\sigma-G_{1} \rho^{2}+G_{2} \rho+G_{3} \\
& \leq-\frac{1+2 G_{1}}{2} \rho^{2}+G_{2} \rho+\frac{2 G_{3}-1}{2} \\
& :=Q(\rho) .
\end{aligned}
$$

Note that condition (58) implies $\left(1+2 G_{1}\right) / 2>0$. In this case, $Q$ has a maximum at $\rho=G_{2} /\left(1+2 G_{1}\right)$. Thus $Q(\rho)<0$ for all real $\rho$ provided

$$
G_{2}^{2} \leq\left(1+2 G_{1}\right)\left(1-2 G_{3}\right), \quad|x|,|y|<1 .
$$

Since $y^{2}<1-x^{2}$, it is left to show that

$$
\begin{aligned}
& \frac{\left(1-B^{2}\right)^{2}}{16(A-B)^{2}} c^{2}\left(1-x^{2}\right) \\
& \quad \leq\left(1+A-B+\kappa(1+B)+\frac{c(1+B)^{2}}{4(A-B)} x\right) \\
& \quad\left(1-A+B-\kappa(-1+B)-\frac{c(1-B)^{2}}{4(A-B)} x\right),
\end{aligned}
$$

$|x|<1$. The above inequality is equivalent to

$$
H(x):=h_{2}(A, B) x+h_{3}(A, B) \geq 0,
$$


where

$$
\begin{aligned}
h_{2}(A, B) & \\
= & \frac{\left(-A\left(B^{2}+1\right)+B\left(3+B^{2}\right)+\left(1-B^{2}\right) B \kappa\right) c}{2(A-B)}, \\
h_{3}(A, B) & (1+A-B+\kappa(1+B))(1-A+B-\kappa(B-1)) \\
& \quad-\frac{\left(1-B^{2}\right)^{2}}{16(A-B)^{2}} c^{2} .
\end{aligned}
$$

Since $|x|<1$, the left-hand side of inequality (76) satisfies

$$
h_{2}(A, B) x+h_{3}(A, B) \geq-\left|h_{2}(A, B)\right|+h_{3}(A, B) .
$$

Now it is evident from (59) that $H(x) \geq 0$ which establishes inequality (76).

Thus $\Psi$ satisfies the hypothesis of Lemma 1, and hence $\operatorname{Re} p(z)>0$, or equivalently

$$
\frac{(A-B) u_{p}^{\prime}+(1-B) z u_{p}^{\prime \prime}}{(A-B) u_{p}^{\prime}-(1+B) z u_{p}^{\prime \prime}} \prec \frac{1+z}{1-z}
$$

By definition of subordination, there exists an analytic selfmap $w$ of $\mathbb{D}$ with $w(0)=0$ and

$$
\frac{(A-B) u_{p}^{\prime}(z)+(1-B) z u_{p}^{\prime \prime}(z)}{(A-B) u_{p}^{\prime}(z)-(1+B) z u_{p}^{\prime \prime}(z)}=\frac{1+w(z)}{1-w(z)} .
$$

A simple computation shows that

$$
1+\frac{z u_{p}^{\prime \prime}(z)}{u_{p}^{\prime}(z)}=\frac{1+A w(z)}{1+B w(z)}
$$

and hence

$$
1+\frac{z u_{p}^{\prime \prime}(z)}{u_{p}^{\prime}(z)} \prec \frac{1+A z}{1+B z} .
$$

Relation (5) also shows that

$$
\frac{z\left(z u_{p}(z)\right)^{\prime}}{z u_{p}(z)}=1+\frac{z u_{p-1}^{\prime \prime}(z)}{u_{p-1}^{\prime}(z)} .
$$

Together with Theorem 10 , relation (83) immediately yields the following result for $z u_{p}(z) \in \mathcal{S}^{*}[A, B]$.

Theorem 11. Let $c$ and $\kappa$ be real numbers such that $(A-$ $B) u_{p-1}^{\prime}(z) \neq(1+B) z u_{p-1}^{\prime \prime}(z)$ for all $z \in \mathbb{D}$ and $-1 \leq B<A \leq 1$. Suppose

$$
\kappa(1+B) \geq \frac{(1+B)^{2}}{4(\mathrm{~A}-B)}|c|-(A-2 B) .
$$

Further let $A, B, \kappa$, and c satisfy

$$
\begin{aligned}
(A- & 2 B+\kappa(1+B))(2 B-A+\kappa(1-B)) \\
\geq & \frac{\left(1-B^{2}\right)^{2}}{16(A-B)} c^{2} \\
& +\left|\frac{B^{3}-(A-B)\left(1+B^{2}\right)+\left(1-B^{2}\right) B \kappa}{2(A-B)} c\right| .
\end{aligned}
$$

Then $z u_{p}(z) \in \mathcal{S}^{*}[A, B]$.

\section{Competing Interests}

The authors declare that they have no competing interests.

\section{References}

[1] W. Janowski, "Some extremal problems for certain families of analytic functions. I," Annales Polonici Mathematici, vol. 28, pp. 297-326, 1973.

[2] R. M. Ali, V. Ravichandran, and N. Seenivasagan, "Sufficient conditions for Janowski starlikeness," International Journal of Mathematics and Mathematical Sciences, vol. 2007, Article ID 62925, 7 pages, 2007.

[3] R. M. Ali, R. Chandrashekar, and V. Ravichandran, "Janowski starlikeness for a class of analytic functions," Applied Mathematics Letters, vol. 24, no. 4, pp. 501-505, 2011.

[4] A. W. Goodman, Univalent Functions, vol. 1-2, Mariner, Tampa, Fla, USA, 1983.

[5] S. S. Miller and P. T. Mocanu, Differential Subordinations, Monographs and Textbooks in Pure and Applied Mathematics, vol. 225, Dekker, New York, NY, USA, 2000.

[6] Á. Baricz, "Geometric properties of generalized Bessel functions," Publicationes Mathematicae Debrecen, vol. 73, no. 1-2, pp. 155-178, 2008.

[7] Á. Baricz, "Geometric properties of generalized Bessel functions of complex order," Mathematica, vol. 48, no. 71, pp. 13-18, 2006.

[8] Á. Baricz and S. Ponnusamy, "Starlikeness and convexity of generalized Bessel functions," Integral Transforms and Special Functions, vol. 21, no. 9-10, pp. 641-653, 2010.

[9] Á. Baricz and R. Szász, "The radius of convexity of normalized Bessel functions of the first kind," Analysis and Applications, vol. 12, no. 5, pp. 485-509, 2014.

[10] V. Selinger, "Geometric properties of normalized Bessel functions," Pure Mathematics and Applications, vol. 6, no. 2-3, pp. 273-277, 1995.

[11] R. Szász and P. A. Kupán, "About the univalence of the Bessel functions," Studia Universitatis Babeș-Bolyai Mathematica, vol. 54, no. 1, pp. 127-132, 2009.

[12] S. S. Miller and P. T. Mocanu, "Differential subordinations and inequalities in the complex plane," Journal of Differential Equations, vol. 67, no. 2, pp. 199-211, 1987. 


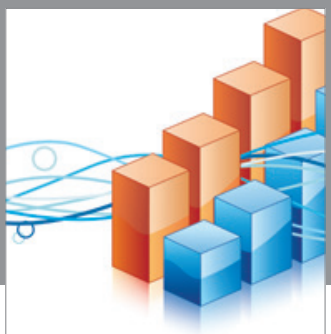

Advances in

Operations Research

vatem alat4

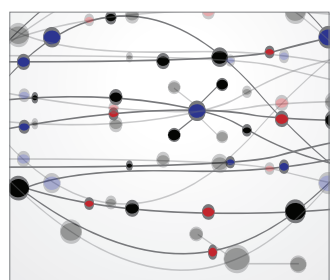

\section{The Scientific} World Journal
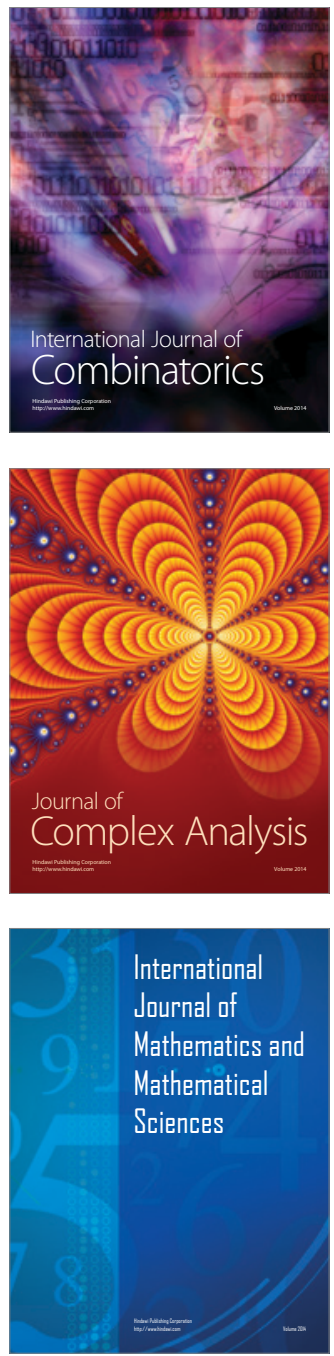
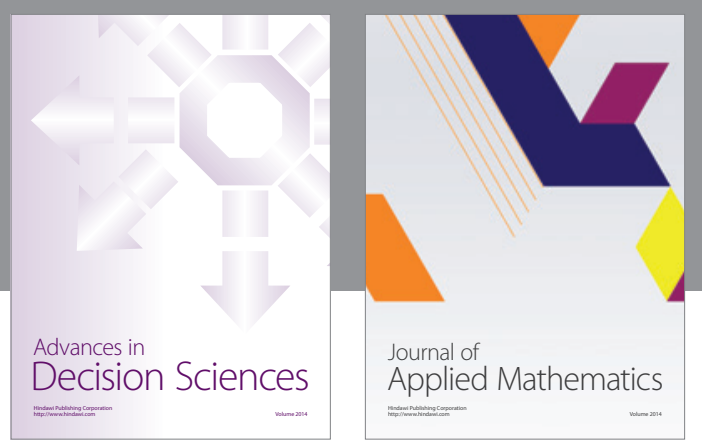

Algebra

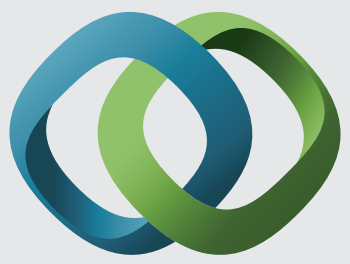

\section{Hindawi}

Submit your manuscripts at

http://www.hindawi.com
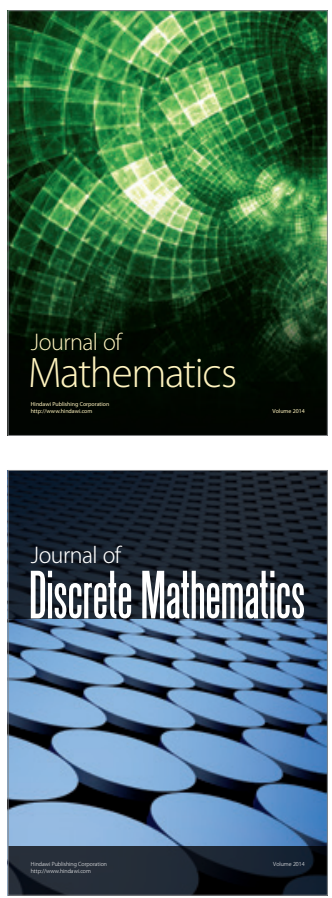

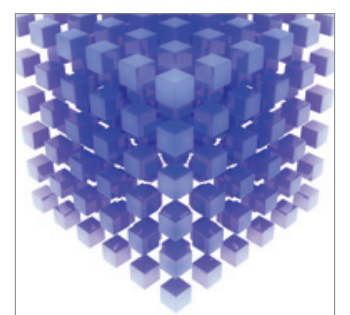

Mathematical Problems in Engineering
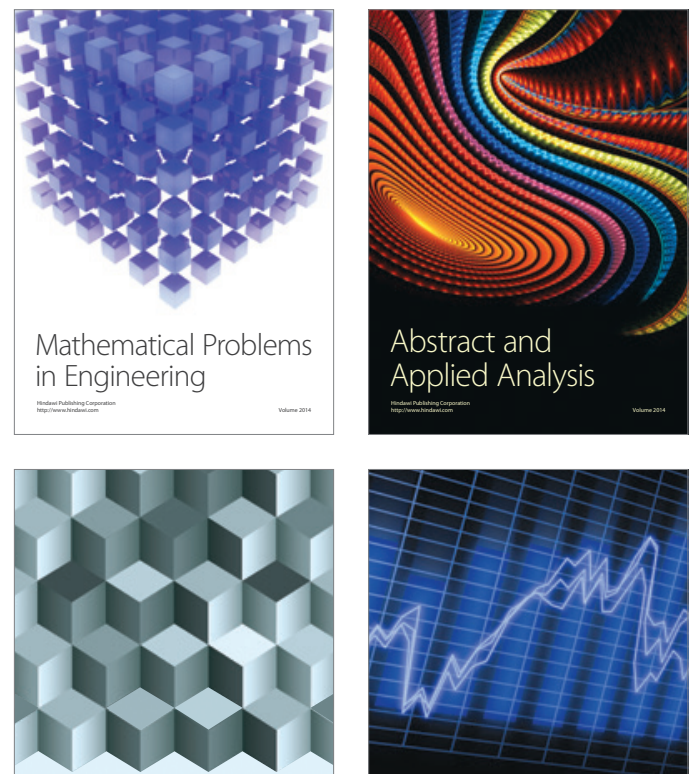

Journal of

Function Spaces

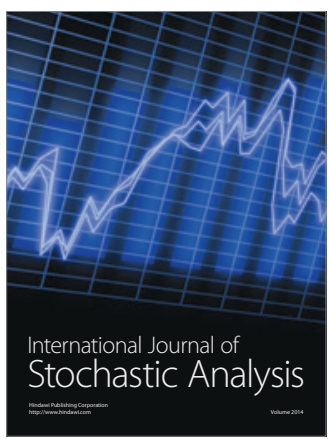

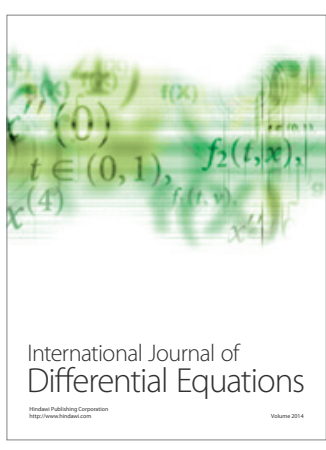
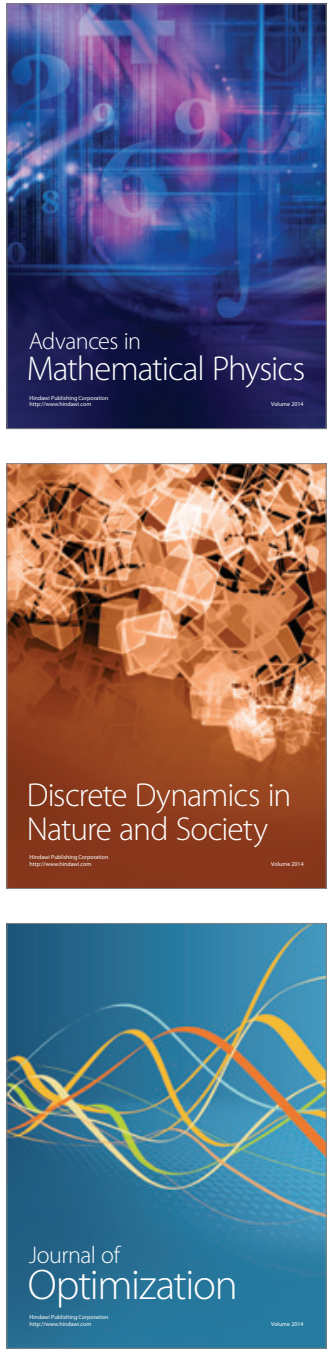